\title{
Improvement of Edge Brightening by Means of Q Factor Minimization in Circular Antenna Apertures: High Efficient Taylor-Like Patterns
}

\author{
AARÓN ÁNGEL SALAS-SÁNCHEZ ${ }^{\circledR 1,2}$, PAOLO ROCCA ${ }^{\circledR}$, (Senior Member, IEEE), \\ JUAN ANTONIO RODRÍGUEZ-GONZÁLEZ ${ }^{\circledR 2}$, (Senior Member, IEEE), \\ M. ELENA LÓPEZ-MARTÍN ${ }^{3}$, AND FRANCISCO J. ARES-PENA ${ }^{\circledR}$, (Fellow, IEEE) \\ ${ }^{1}$ ELEDIA@UniTN, DISI, University of Trento, 38123 Trento, Italy \\ ${ }^{2}$ Department of Applied Physics, Faculty of Physics, CRETUS Institute, University of Santiago de Compostela, \\ Campus Vida s/n, E-15782 Santiago de Compostela, Spain \\ ${ }^{3}$ Department of Morphological Sciences, Faculty of Medicine, CRETUS Institute, University of Santiago de Compostela, \\ C/San Francisco s/n, E-15782 Santiago de Compostela, Spain \\ Corresponding author: Francisco J. Ares-Pena (francisco.ares@usc.es)
}

This work was supported in part by the FEDER/Ministerio de Ciencia, Innovación y Universidades-Agencia Estatal de Investigación, under Project TEC2017-86110-R. The work of Aarón A. Salas-Sánchez was supported by the Xunta de Galicia under the Postdoctoral Fellowship with ref. ED481B 2018/008.

\begin{abstract}
Implications and improvements of edge brightening effects led by $Q$ factor minimization restricted to keep the same level of directivity for high efficiency continuous circular aperture distributions are here reported. In this manner, an optimization strategy for a minimum $Q$ value -keeping the same level of efficiency and restricting the maximum sidelobe level $(S L L)$ - is envisaged. As application of the method, a design procedure devoted to reduce the $Q$ factor of the antenna aperture distributions while keeping a high level of efficiency is outlined. Then, these optimal Taylor distributions are used as initial point to develop an optimization strategy. This procedure is devoted to search Taylor-like distributions which offer a good compromise between low $Q$ factor and high efficiency values with potentials for the antenna design scenario, based on a decrease in edge brightening effects led by the minimization of the aforementioned $Q$ ratio.
\end{abstract}

INDEX TERMS Circular Taylor distributions, edge brightening, optimization techniques, $Q$ factor, super gain ratio.

\section{INTRODUCTION}

The presence of edge brightening effects in continuous aperture distributions introduces problems in terms of practical realization of different array patterns [1].

Additionally, parameters as directivity must be taken into account into a synthesis procedure for improving the performance of these design techniques.

Alternatively, the so-called $Q$ factor represents a crucial parameter in the antenna design arena. More concretely, in [2], Chu established the basis of the theoretical description of the $Q$ factor concept for an omni-directional antenna. It was defined as the ratio between the stored energy and the radiated energy and exploiting in the analysis of the physical limitations of the antenna in terms of bandwidth versus gain.

The associate editor coordinating the review of this manuscript and approving it for publication was Yanhui Liu ${ }^{\mathbb{D}}$.
From this milestone, the small antennas framework started to attract the attention of the researchers and authors as Rhodes faced this topic trying to adapt these new possibilities to different scenarios. More concretely, he focused on planar antennas [3] and also he analyzed the stored energy term of planar apertures [4]. Another foundational work that deserves attention is the one developed by Hansen in 1981 [5], where the $Q$ factor concept has been discussed to review the limitations of different types of antennas. In line with these seminal developments of Rhodes and Hansen, Thiele et al. [6] devised an alternative formal approach for predicting the realizable radiation $Q$ of an electrically small antenna. Also, another example can be found in the work of Jonsson et al. [7], where the $Q$ factor bounds for super-directive antennas have been analyzed by using an example with electrical size $k a=0.51$ (where $k$ is the wavenumber and $a$ the radius of the minimum sphere that contains the antenna-also known as the 
Chu radius [2]-). Then, recent examples as the interesting review made by Schab et al. [8] have been devoted to compare the impact and the determination of this parameter within the radiation properties of the antenna systems. Another interesting and recent approach was discussed by Gustafsson and Capek [9] and it represents a deep and careful study about a trade-off between gain and $Q$ factor for antenna systems.

Other studies regarding infinite planar phased arrays have been conducted. For instance, Kwon and Pozar [10] reported antenna configurations involving arrays of dipoles with lengths from $0.15 \lambda$ to $0.45 \lambda$. In the same line, but generalizing the study to a wider range of dipole lengths and overcoming the limit $k a=1$, more recent approaches can be found in the works of Ludvig-Osipov and Jonsson [11], [12]. Here, they calculated the $Q$ factor and compared their results with MoM simulations in the framework of two-dimensional periodic arrays for both scenarios with and without ground plane.

Considering these descriptions, but focusing on the standard array pattern synthesis scenario and more concretely on its application to cases where superdirectivity effects are moderately low, we can translate the $Q$ factor of the distribution as the ratio between the expression of the radiated far field and the expression of the field within the antenna [13]. Then, the understanding of the $Q$ factor by means of the super gain ratio relation [14], [15] suggests that this parameter can give us an idea about the level of inefficiency of the finite analytical aperture distribution to reproduce the infinite pattern distribution. In addition and diversely to the cases referred in the standard literature of small antennas (i.e., with $k a<1$ ), improvements on different radiation characteristics are here reported by reducing the levels of $Q$. Consequently, due to the range of application that we are proposing, the values here analyzed will be less than the unit. Thus, in this framework, by following the definition of this parameter, an improvement in terms of edge brightening effects led by a decrease in this $Q$ factor is here reported, which is highly interesting in terms of practical realization of the distribution.

However if, on the one hand it is important to reduce the effect as the edge brightening, on the other hand it is also fundamental not to provoke critical reductions of the antenna directivity. Due to this reason, a search devoted to reach a compromise between directivity and $Q$ factor is mandatory.

Examples of this edge brightening alleviation without exploiting the $Q$ factor concept are reported in [16] and [17]. In [16], the problem of minimizing edge brightening effects was faced by filling nulls into the array pattern generated by the distribution. As a drawback, although a multiplicity of solutions has been reached, the generation of a complex aperture distribution and a loss in directivity -due to the null filling - can be highlighted. Alternatively, a direct minimization of the edge brightening phenomenon has been performed in [17]. In this case, although a best level of this parameter could be reached, the $\mathrm{Q}$ factor strategy will assure a monotonic behavior of the aperture that also can be highlighted as interesting thinking about practical realization. Until now, no relation between $Q$ factor and this performance in the border of the aperture has been analyzed.

So, the idea here proposed of a reduction in the $Q$ factor of the continuous aperture distribution -by keeping the same efficiency/directivity level- is linked to an energy decreasing into the invisible region. This fact uniquely can be understood as a reduction of the distribution peaks at the edge of the aperture (i.e. the edge brightening effect [18]) in comparison with the reference case, as well as a smoother transition of the excitation at the end of its tail. Thus, the extreme variability of this continuous aperture will be minimized as a result and a smoother transition (mainly near the edge) has to be expected. In this manner, a more stable aperture regarding errors or bandwidth will be produced. So, based on the energy considerations and restrictions abovementioned, the minimization of these parameters of the aperture would make the antenna (without any kind of bound to a concrete size requirement) a more efficient radiation system [19].

Therefore, by focusing on this idea, a procedure keeping the optimal directivities of circular Taylor distributions [20] and improving the $Q$ factor for generating pencil, also called sum, beams is here depicted. Circular Taylor distributions will play a main role in this work, because they represent a direct approach to obtain a pattern of maximum efficiency for a fixed sidelobe level (SLL) requirement. As it is well-known, these analytical distributions can be synthesized to obtain array excitations for reproducing similar patterns from an antenna array [21].

In this work, analytical Taylor distributions are defined by means of suitable optimization strategies in order to minimize the antenna $Q$ factor by also guaranteeing a high efficiency and desired $S L L$ values. The main novelties of this contribution are as follows: 1) a closed-form equation by means of a well-defined finite integral of the $Q$ factor parameter for circular aperture distributions so as to yield a less complex evaluation (with high potentials, if we mainly think about introducing this property in optimization procedures); 2) the definition of a robust optimization method for obtaining high efficient distributions which also present improved edge brightening effects.

Towards this end, the paper is organized as follows. In section II, the theoretical bases of the method are stated. Then, a description of the optimization procedures for $Q$ factor values of the distribution by guaranteeing a specified $S L L$ and a certain directivity is included and discussed in section III. Section IV is devoted to show the results of the optimization methodology here devised by means of circular Taylor patterns and by outlining the advantages in terms of dynamic range ratio $\left(\left|I_{\max } / I_{\min }\right|\right)$ and edge brightening $(E B)$ of the distributions. Finally, some conclusions and remarks are drawn in section $\mathrm{V}$.

\section{THEORETICAL BASIS}

\section{A. Q FACTOR AND DIRECTIVITY}

Let us consider a general circular aperture distribution with a radius $a$ (Fig. 1) which generates a radiation pattern $F(\theta, \phi)$. 


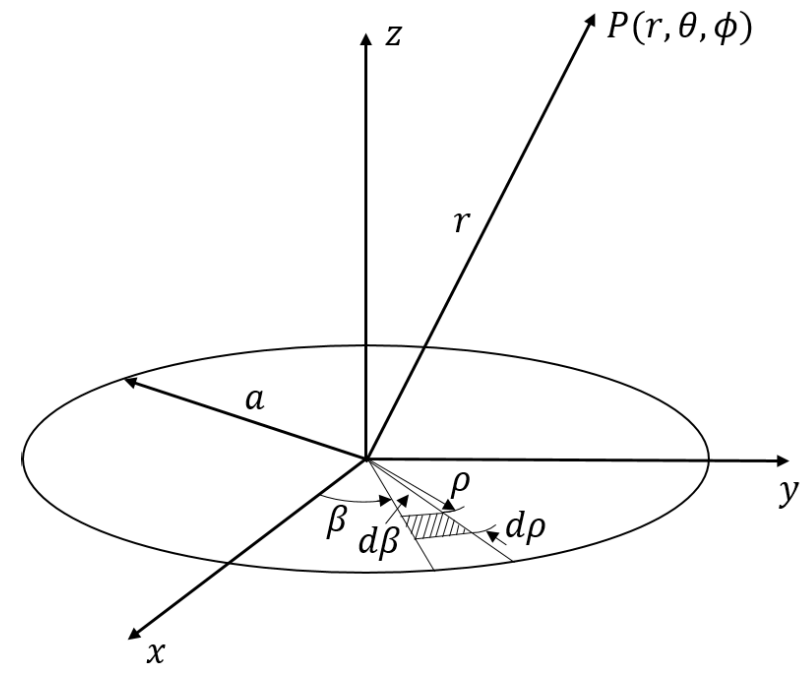

FIGURE 1. Circular aperture $K(\rho, \beta)$ for generating a radiation far field pattern.

On this basis, circular symmetry is assumed and the far field pattern generated by the continuous aperture distribution is rotationally symmetric. In this case, the $Q$ factor can be defined -in a parallelism with the linear case [14], [15]- as the ratio between the reactive power (which is related with the integral of the pattern function in the invisible region) and the radiated power of the antenna (related with this same integral but in the visible region). Therefore, generalizing the analytical expression of the $Q$ factor, for this special case in 2 dimensions, is as follows:

$$
Q=\frac{\int_{0}^{2 \pi} \int_{2 a / \lambda}^{\infty}|F(u, \phi)|^{2} u d u d \phi}{\int_{0}^{2 \pi} \int_{0}^{2 a / \lambda}|F(u, \phi)|^{2} u d u d \phi},
$$

where $u=(2 a / \lambda) \sin (\theta)$.

For simplicity, and also to facilitate the management of this quantity in the optimization process, an alternate closed-form of the expression turns to be convenient. This can be done by introducing in the formalism the concept of superdirectivity (traditionally understood as supergain) and reported in the literature by means of the Super Gain Ratio (SGR) [6], [14], [15]. This $S G R$ is related to the $Q$ factor for simple sources [5] as

$$
Q=S G R-1,
$$

where the $S G R$ is defined as the ratio between the specific directivity and the effective directivity of a given aperture distribution.

In turn, the specific directivity ([14], [15]) -which also can be identified as normalized peak directivity (p. 261, [12]) or simply the aperture illumination efficiency [22] - can be defined as the ratio of the directivity of the aperture and the directivity of a uniformly illuminated aperture. More specifically, the directivity of a circular distribution can be defined as the ratio of the power density in the direction of interest to the average power radiated. So, by assuming isotropic elements and considering that the expression of the directivity of a circular aperture of radius $a$ uniformly illuminated is $D_{0}=4 \pi^{2} a^{2} / \lambda^{2}$ (p. 261, [21]), the specific directivity can be defined as

$$
G=D_{i} / D_{0}=\frac{\lambda^{2}\left|F\left(\theta_{m}, \phi_{m}\right)\right|^{2}}{\pi a^{2} \int_{0}^{2 \pi} \int_{0}^{\pi / 2}|F(\theta, \phi)|^{2} \sin \theta d \theta d \phi}
$$

where $F\left(\theta_{m}, \phi_{m}\right)$ is the value of the pattern function in its maximum point.

According to [23], for aperture sizes with $a \geq 5 \lambda$, it could be accurately approximated (a detailed discussion can be also found in the appendix of the present paper) by:

$$
G=\frac{\lambda^{2}\left|F\left(\theta_{m}, \phi_{m}\right)\right|^{2}}{\pi a^{2} \int_{0}^{2 \pi} \int_{0}^{2 a / \lambda}|F(\sin \theta, \phi)|^{2} \sin \theta d(\sin \theta) d \phi} .
$$

Otherwise, the effective directivity is the specific directivity when the aperture size tends to infinity ([14], [15]). So, let us define as

$$
G_{\infty}=\frac{\lambda^{2}\left|F\left(\theta_{m}, \phi_{m}\right)\right|^{2}}{\pi a^{2} \int_{0}^{2 \pi} \int_{0}^{\infty}|F(\sin \theta, \phi)|^{2} \sin \theta d(\sin \theta) d \phi} .
$$

Then, by exploiting the properties of the Fourier transformation procedure which leads the relationship between the radiation pattern and the aperture distribution, we can express the infinite integral of the pattern function by means of the finite integral of its circular aperture distribution. This expression is the so-called Parseval's formula [15]

$$
\begin{aligned}
& \int_{0}^{2 \pi} \int_{0}^{\infty}|F(\sin \theta, \phi)|^{2} \sin \theta d(\sin \theta) d \phi \\
&=\left(\frac{2 a}{\lambda}\right)^{2} \int_{0}^{2 \pi} \int_{0}^{\pi}|g(p)|^{2} p d p d \phi .
\end{aligned}
$$

where $g(p)$ is the antenna aperture distribution which generates the far field radiation power pattern $F(\theta, \varphi)$, and $p=$ $\pi \rho / a$ (and $\rho \in[0, a]$ ). This expression of the aperture is related with the circular aperture shown in Fig. 1 by the formula (p. 214, [21])

$$
g(p)=\frac{2 a^{2}}{\pi} K(a p / \pi, \beta)
$$

where $K(a p / \pi, \beta)=K(a p / \pi)$ due to the symmetry of the problem.

Therefore, a compact expression of the $S G R$-as it was aforementioned, defined as the ratio between the specific directivity and the effective directivity of a given aperture distribution ([14], [15])- is as follows:

$$
S G R=G / G_{\infty}=\frac{\left(\frac{2 a}{\lambda}\right)^{2} \int_{0}^{2 \pi} \int_{0}^{\pi}|g(p)|^{2} p d p d \phi}{\int_{0}^{2 \pi} \int_{0}^{2 a / \lambda}|F(u)|^{2} u d u d \phi}
$$




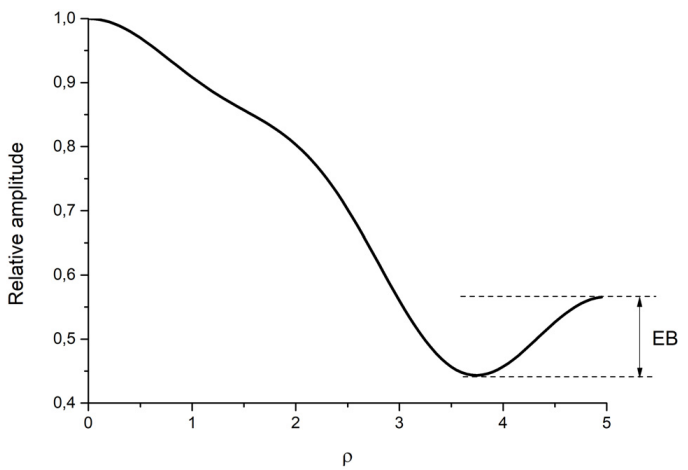

FIGURE 2. Graphical description of the determination of the parameter devoted to evaluate the effect of edge brightening (EB) in the continuous aperture distribution $g(\pi \rho / \boldsymbol{a})$. Case in example: relative aperture of a circular Taylor distribution with $\bar{n}=5, S L L_{d}=-25 d B$.

and, accordingly, the $Q$ factor is determined through Eq. (1) by means of a formulation based on finite integrals

$$
Q=\frac{\left(\frac{2 a}{\lambda}\right)^{2} \int_{0}^{2 \pi} \int_{0}^{\pi}|g(p)|^{2} p d p d \phi}{\int_{0}^{2 \pi} \int_{0}^{2 a / \lambda}|F(u)|^{2} u d u d \phi}-1
$$

\section{B. EDGE BRIGHTENING}

The edge brightening [1], [16], [17] can be defined as a pronounced rise in amplitude at the boundary of the aperture distribution. So, in order to evaluate the impact of the edge brightening effect, let us mathematically define a quality parameter $E B$. To determine this quality parameter, the difference between the level of amplitude in the border of the aperture distribution and the local minimum previous to this value (in this same amplitude curve) can be proposed (Fig. 2).

Therefore, the proposed definition is

$$
E B=g(\pi)-g\left(p_{\min }\right),
$$

where $p_{\min }$ is the local minimum previous to the edge of the continuous aperture distribution $(p=\pi)$

\section{CIRCULAR TAYLOR DISTRIBUTION}

As it was above mentioned, the present work is focused on the use of circular Taylor distributions due to their high efficiency.

The basis of this approach is the modification of the roots of the uniform distribution, it is, when the aperture is uniformly excited. This represents the most directive distribution for a given radius $a$ of a circular antenna. The expression of the radiation far field pattern in the uniform case becomes [21]:

$$
F(u)=\frac{J_{1}(\pi u)}{\pi u}
$$

where $u=(2 a / \lambda) \sin \theta$ and $J_{1}(\pi u)$ is the Bessel function of first order.

More concretely, the method devised by Taylor for continuous circular aperture distributions is based on altering a number of $\bar{n}-1$ roots of the pattern and controlling the level of the innermost $\bar{n}-1$ ring side lobes [20]. Thus, the sum pattern produced by the circular Taylor distribution would be given by [20], [21]:

$$
F(u)=\frac{J_{1}(\pi u)}{\pi u} \cdot \frac{\prod_{n=1}^{\bar{n}-1}\left(1-\frac{u^{2}}{u_{n}^{2}}\right)}{\prod_{n=1}^{\bar{n}-1}\left(1-\frac{u^{2}}{\gamma_{1, n}^{2}}\right)},
$$

where $\gamma_{1, n}$ is the $\mathrm{n}$-th root of the Bessel function of first order $\left[J_{1}\left(\gamma_{1, n} \pi\right)=0\right]$ and $u_{n}, \mathrm{n}=1, \ldots, \mathrm{N}$ the manipulated roots of the pattern by means of this methodology are

$$
u_{n}^{2}=\gamma_{1, n}^{2} \frac{A^{2}+\left(n-\frac{1}{2}\right)^{2}}{A^{2}+\left(\bar{n}-\frac{1}{2}\right)^{2}},
$$

where $A$ is related with the desired sidelobe level $\left(S L L_{d}\right)$, through the expression $S L L_{d}=-20 \log _{10}(\cosh \pi A)$.

Finally, the expression of the continuous aperture which generates the pattern is [20], [21]

$$
g(p)=\frac{2}{\pi^{2}} \sum_{m=0}^{\bar{n}-1} \frac{F\left(\gamma_{1, m}\right)}{J_{0}^{2}\left(\gamma_{1, m} \pi\right)} J_{0}\left(\gamma_{1, m} p\right)
$$

where $p=\pi \rho / a, \rho$ is the radial coordinate of the aperture, so it is in the range $\rho \in[0, a]$ and $J_{0}\left(\gamma_{1, m} \pi\right)$ is the Bessel function of the first kind and order zero, evaluated in the roots of the Bessel function of the first order.

\section{OPTIMIZATION METHOD}

In this section, the formulation highlighted in the previous section is exploited within an optimization strategy devoted to afford low $Q$ factor levels by guaranteeing high efficiencies for a desired $S L L$. The proposed optimization process can be understood as a single objective problem (SOP) [24] and it can be summarized as follows:

1. As starting point, a circular Taylor pattern is established by selecting the $\bar{n}$ value which brings the maximum efficiency for a certain $S L L . u_{n}^{0}$ denotes the roots of this pattern.

2. Small perturbations $\delta \vec{u}$ to these initial roots are introduced. The main objective of these perturbations is to synthesize a pattern in which the efficiency is maximum, the $Q$ factor is low and the $S L L$ is controlled.

First of all, it can be concluded that there are three objectives to be considered in the design concerning the efficiency, $Q$ factor, and $S L L$. Moreover, the optimization variables are continuous and real.

In this process, the following scalar cost function is minimized:

$$
C(\delta \vec{u})=c_{1} \cdot f_{S L L}+c_{2} \cdot\left|\eta_{\text {Taylor }}-\eta\right|+c_{3} \cdot Q
$$

where $\eta$ denotes the aperture illumination efficiency (3), $\eta_{\text {Taylor }}$ is the aperture illumination efficiency of the reference aperture: the initial Taylor aperture distribution, $Q$ is the quality factor (expressed in natural units), whereas $c_{i}$ are the weights to control the importance given to each term 


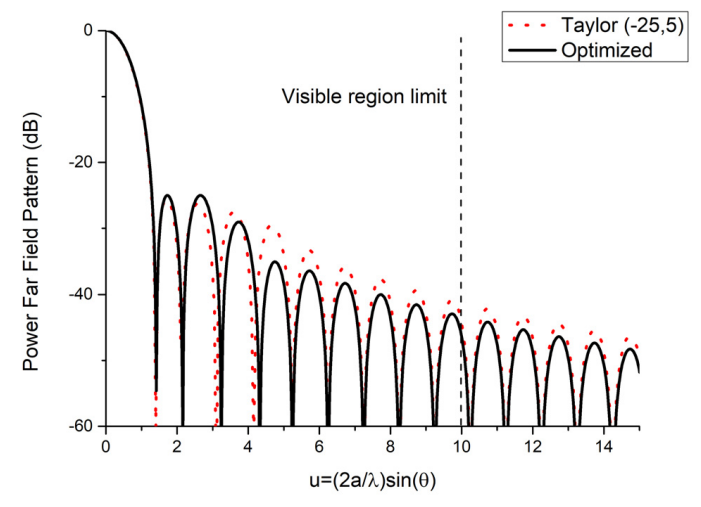

FIGURE 3. Circular Taylor pattern with $\bar{n}=5, S L L_{d}=-25 d B$ and optimized pattern by considering a loss in efficiency of $0 \%$ with respect to this circular Taylor pattern.

of (15). The function $f_{S L L}$, which penalizes the increasing in the sidelobe level, is defined by

$$
f_{S L L}= \begin{cases}\left(S L L_{0}-S L L_{d}\right)^{2}, & \text { if } S L L_{0} \geq S L L_{d} \\ 0, & \text { otherwise }\end{cases}
$$

where $S L L_{0}$ and $S L L_{d}$ are, respectively, the obtained and the desired sidelobe level of the pattern. The squared difference is here adopted for amplifying the error of this term. In such a way, the biggest error gets amplified more and, in consequence, it will obtain more priority for improvement. Moreover, $\delta \vec{u}=\left[\delta u_{1}, \delta u_{2}, \ldots, \delta u_{\bar{n}-1}\right]^{T}$ is the vector of perturbations applied to the roots of the initial circular Taylor pattern.

To develop this procedure, a hybrid approach described on section 3 of [25], which is based on the procedure developed in [26] and involves the Simulated Annealing (SA) algorithm [27] with a modification based on the Downhill Simplex (DS) method [28] has been implemented. More concretely, as it is referred in [25], the procedure devoted to found the minimum is equal to the one developed by DS with the following main difference: A $T$ parameter analogous to the temperature for the SA algorithm [27] is introduced. In such a way, a probabilistic component (modeled exactly in the same manner as in the SA) is added in the DS strategy for comparing with the other values of the cost function represented in the Simplex [28]. The aforementioned $T$ is set in order to explore a wider space of solutions. In this manner, the algorithm improves the searching and prevents to always fall in the same local minimum. In our case, we set $T$ as 100 .

\section{RESULTS}

Let us first consider the case of a circular Taylor-like pattern with a $S L L_{d}$ of $-25 \mathrm{~dB}$. So, in order to proceed with the optimization of the $Q$ factor and improve the solution in terms of edge brightening for distributions with a high efficiency, a circular Taylor pattern distribution with $\bar{n}=5$ has been chosen (see Figs. 3 and 4). The initial roots, as well as the directivity, the $S L L$, the $Q$ factor, the dynamic range ratio $\left(\left|I_{\max } / I_{\min }\right|\right)$ and the edge brightening quality value $(E B)$ of this pattern are shown in Table 1.

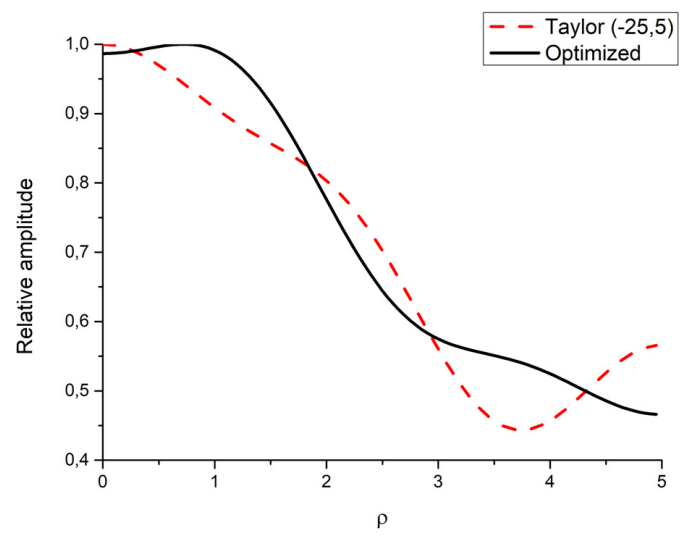

FIGURE 4. Relative amplitudes which generate the regular Taylor case with $\bar{n}=5, S L L_{d}=-25 d B$ and the optimized one of the Fig. 3.

TABLE 1. Roots, SLL, Directivity, $Q$ Factor, Dynamic Range Ratio and Edge Brightening of Taylor Pattern with $\bar{n}=5$ and $S L L_{d}=-25 d B$ and the pattern with optimized $Q$ value.

\begin{tabular}{ccc}
\hline \hline $\begin{array}{c}\text { Radius of the } \\
\text { aperture distribution }\end{array}$ & $\begin{array}{c}\text { Taylor pattern } \\
\bar{n}=5 \lambda\end{array}$ & Optimized pattern \\
\hline$u_{1}$ & 1.4026 & \\
$u_{2}$ & 2.1258 & 1.4118 \\
$u_{3}$ & 3.1021 & 2.1543 \\
$u_{4}$ & 4.1568 & 3.2395 \\
$S L L(\mathrm{~dB})$ & -25.52 & 4.3297 \\
\hline \hline Directivity $(\mathrm{dBi})$ & 29.66 & -25.00 \\
$Q(\mathrm{~dB})$ & -17.62 & 29.66 \\
$\left|I_{\max } / I_{\min }\right|$ & 2.25 & -19.53 \\
$E B$ & 0.1917 & 2.15 \\
\hline \hline
\end{tabular}

The optimization process was initialized by considering these roots as starting solution. Several cases have been run in which the weight of the efficiency term $-c_{2}$ coefficient in (15)- has been varied in order to find a good compromise between high efficiency and low $Q$ factor values. These coefficients have been set in all the examples here analyzed as $c_{1}=1200, c_{2}=1500$, y $c_{3}=1000$. Results keeping the same level of efficiency (i.e., having an efficiency loss of $\Delta \eta=\eta_{\text {Taylor }}-\eta=0 \%$ ) and consequently the directivity of the circular Taylor pattern distribution are shown in Table 1. It can be observed that, by keeping the same efficiency, this method led a decrease of the $Q$ factor of $1.91 \mathrm{~dB}$ (a $35.61 \%$ in natural units) and both the dynamic range ratio and the edge brightening were reduced a $4.44 \%$ and a $18.68 \%$, respectively. The relative amplitude of both apertures (the initial and the optimized) are shown (Fig. 4) as well as a comparison between the generated radiation far field pattern and the regular Taylor case (Fig. 3). Finally, in order to complete the discussion, the roots which generate each one of the patterns can be found in Table 1 with a report of the resulting quality parameters.

For illustrating the behavior of the method and to understand how it works in case of different side lobe topography, $S L L_{d}$ of $-30 \mathrm{~dB}$ and $-35 \mathrm{~dB}$ are also analyzed. 


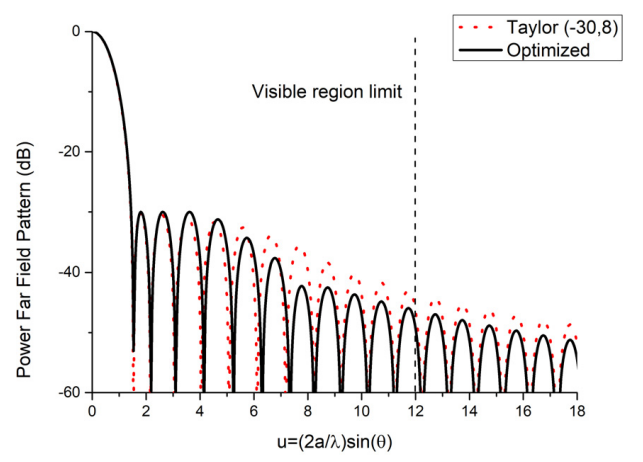

FIGURE 5. Circular Taylor pattern with $\bar{n}=8, S L L_{d}=-30 d B$ and optimized pattern by considering a loss in efficiency of $0 \%$ with respect to this circular Taylor pattern.

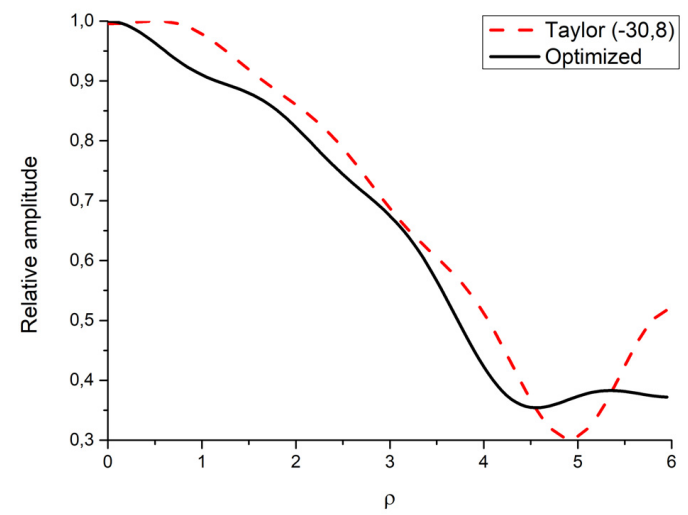

FIGURE 6. Relative amplitudes which generate the regular Taylor case with $\bar{n}=8, S L L_{d}=-30 d B$ and the optimized one of the Fig. 5 .

In the case of a $S L L_{d}$ of $-30 \mathrm{~dB}$, the most efficient choice is the circular Taylor pattern distribution with $\bar{n}=8$ (see Figs. 5 and 6). In the same manner that in the previous case, the initial roots, as well as the $S L L$, the directivity, the $Q$ factor, the dynamic range ratio $\left(\left|I_{\max } / I_{\min }\right|\right)$ and the edge brightening quality value $(E B)$ are shown in Table 2 .

To outline the performance of the method in terms of aperture distribution, the relative amplitude of both cases (the initial and the optimized continuous aperture distributions) are shown in Fig. 6. A comparison between the two radiation far-field patterns is also shown in Fig. 5, as well as the roots of each one of them (see Table 2 ).

Additionally, for the case of a circular Taylor pattern distribution of $S L L_{d}=-35 \mathrm{~dB}$, a $\bar{n}=13$ has been chosen (see Figs. 7 and 8) for guaranteeing a high efficiency.

The initial roots, the actual $S L L$ as well as the directivity, the $Q$ factor, the dynamic range ratio $\left(\left|I_{\max } / I_{\min }\right|\right)$ and the edge brightening quality value $(E B)$ of the compared patterns are shown in Table 3.

In the same spirit of the previous analysis, the performance of the method is illustrated in Figs. 7 and 8, where both apertures (the initial and the optimized ones) as well as the radiation far field patterns are shown. The roots related with the generated patterns in this case are reported with the results in Table 3.
TABLE 2. Roots, SLL, Directivity, Q Factor, Dynamic Range Ratio and Edge Brightening of Taylor Pattern with $\bar{n}=8$ and $S L_{d}=-30 d B$ and the pattern with optimized $Q$ value.

\begin{tabular}{|c|c|c|}
\hline $\begin{array}{c}\text { Radius of the } \\
\text { aperture distribution } \\
\qquad a=6 \lambda\end{array}$ & $\begin{array}{c}\text { Taylor pattern } \\
\bar{n}=8 \\
S L L_{d}=-30 \mathrm{~dB}\end{array}$ & Optimized pattern \\
\hline$u_{1}$ & 1.5283 & 1.5385 \\
\hline$u_{2}$ & 2.1634 & 2.1814 \\
\hline$u_{3}$ & 3.0610 & 3.0988 \\
\hline$u_{4}$ & 4.0502 & 4.1462 \\
\hline$u_{5}$ & 5.0776 & 5.2422 \\
\hline$u_{6}$ & 6.1242 & 6.3023 \\
\hline$u_{7}$ & 7.1815 & 7.3423 \\
\hline$S L L(\mathrm{~dB})$ & -30.24 & -30.00 \\
\hline Directivity (dBi) & 30.98 & 30.98 \\
\hline$Q(\mathrm{~dB})$ & -18.66 & -21.18 \\
\hline$\left|I_{\max } / I_{\min }\right|$ & 3.30 & 2.82 \\
\hline$E B$ & 0.1899 & 0.1459 \\
\hline
\end{tabular}

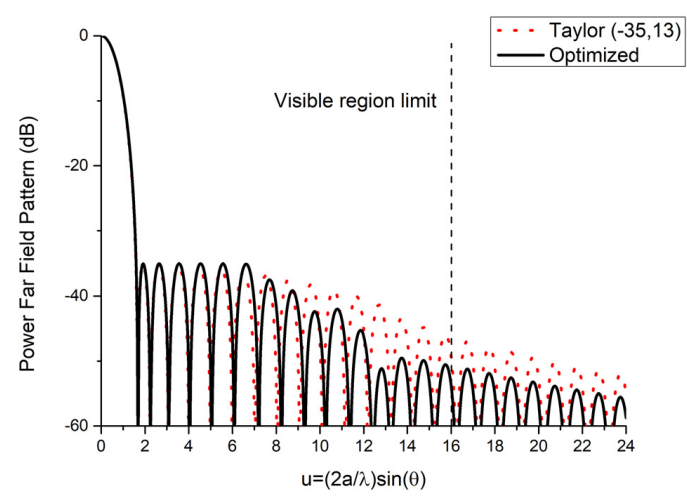

FIGURE 7. Circular Taylor pattern with $\bar{n}=13, S L L_{d}=-35 d B$ and optimized pattern by considering a loss in efficiency of $0 \%$ with respect to this circular Taylor pattern.

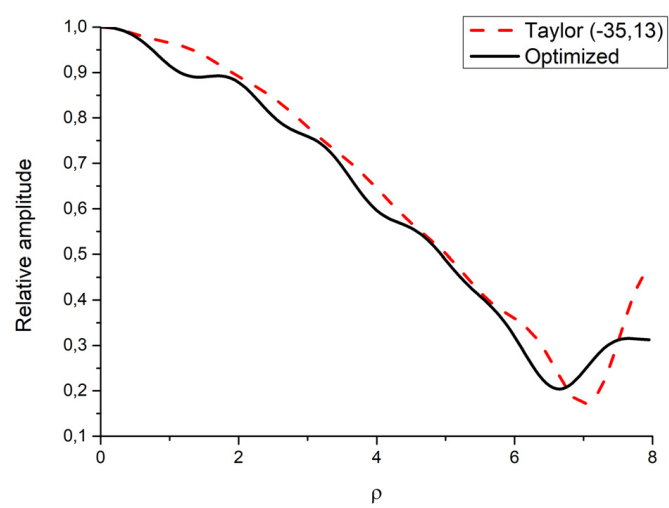

FIGURE 8. Relative amplitudes which generate the regular Taylor case with $\bar{n}=13, S L L_{d}=-35 d B$ and the optimized one of the Fig. 7 .

Results constrained to an efficiency loss with respect to the circular Taylor pattern of $\Delta \eta=\eta_{\text {Taylor }}-\eta=0 \%$ for each $S L L(-30 \mathrm{~dB}$ and $-35 \mathrm{~dB})$ are respectively shown in Tables II and III. It can be observed that, by keeping the same level of efficiency, this method led a decrease of the $Q$ factor of $2.52 \mathrm{~dB}$ (a $44.01 \%$ in natural units) and 
TABLE 3. Roots, SLL, Directivity, Q Factor, Dynamic Range Ratio and Edge Brightening of Taylor Pattern with $\bar{n}=13$ and $S L L_{d}=-35 d B$ and the pattern with optimized $Q$ value.

\begin{tabular}{ccc}
\hline \hline $\begin{array}{c}\text { Radius of the } \\
\text { aperture distribution }\end{array}$ & $\begin{array}{c}\text { Taylor pattern } \\
\bar{n}=13\end{array}$ & Optimized pattern \\
$a=8 \lambda$ & $S L L_{d}=-35 d B$ & \\
\hline \hline$u_{1}$ & 1.6669 & 1.6775 \\
$u_{2}$ & 2.2334 & 2.2495 \\
$u_{3}$ & 3.0694 & 3.0926 \\
$u_{4}$ & 4.0080 & 4.0439 \\
$u_{5}$ & 4.9921 & 5.0471 \\
$u_{6}$ & 5.9993 & 6.0896 \\
$u_{7}$ & 7.0198 & 7.1962 \\
$u_{8}$ & 8.0484 & 8.2358 \\
$u_{9}$ & 9.0824 & 9.2995 \\
$u_{10}$ & 10.1202 & 10.2580 \\
$u_{11}$ & 11.1606 & 11.3664 \\
$u_{12}$ & 12.2031 & 12.4207 \\
\hline$S L L(\mathrm{~dB})$ & -35.11 & -35.00 \\
Directivity $(\mathrm{dBi})$ & 33.15 & 33.15 \\
$Q(\mathrm{~dB})$ & -19.81 & -23.28 \\
$\left|I_{\max } / I_{\min }\right|$ & 5.82 & 4.90 \\
$E B$ & 0.1952 & 0.1384 \\
\hline \hline
\end{tabular}

of $3.47 \mathrm{~dB}$ (a $55.02 \%$ in natural units). In case of dynamic range ratio, the optimized solutions have reached a reduction of a $14.55 \%$ and a $15.81 \%$ (a $1.26 \%$ and $11.37 \%$ less than in the $-25 d B$ case, respectively). Regarding edge brightening effects, they have been reduced a $23.17 \%$ and a $29.10 \%$ on each case. They represent improvements of a $4.49 \%$ and a $10.42 \%$ respectively. So, we confirm how the method tends to alleviate the tendency of the aperture of impinging a much higher level of excitation on its edges than in the medium part of its tail. These results only can be understood due to the fact that a lower $Q$ is related to a decreasing in the level of far field power radiation pattern present in the visible region limit. Therefore, from the inspection of Figs. 4, 6 and 8 it can be stated that generally improvements in terms of $Q$ imply a smooth transition on the tail of the aperture distribution in comparison with the initial case.

\section{v. CONCLUSION}

In this paper, several numerical optimizations were performed and results of improving the $Q$ factor by preventing efficiency losses have been shown. A relation with the edge brightening (and also the dynamic range ratio) of the distribution has been established and, in this manner, the practical applicability of this process is devised. The minimization of the $Q$ ratio -by keeping the same directivity level- within the far field power generated by an aperture distribution is translated in a aperture distribution with less edge brightening and -generally- on a less dynamic range ratio of the aperture. More concretely, for a $S L L_{d}=-25 d B$, an improvement of a $35.61 \%$ of $Q$ factor leads improvements in terms of dynamic range ratio $(4.44 \%)$ and edge brightening $(18.68 \%)$. At the same time, for a $S L L_{d}=-30 d B$, through an improvement of a $44.01 \%$ of $Q$ factor, the improvement in terms of dynamic range ratio and edge brightening were $14.55 \%$ and $23.17 \%$, respectively. Finally, for a level of $S L L_{d}=-35 d B$, an improvement of $Q$ about $55.02 \%$ leads improvements of $15.81 \%$ and $29.10 \%$ in terms of edge brightening of the continuous aperture distribution. Based on these results, a method to design good distributions devoted to afford high efficiency and improving the aperture variability (by reducing the $Q$ factor for a required directivity) was developed. This technique shows interesting potentials for the antenna design discipline. At the same time, this strategy overcomes the results of standard techniques -based on a direct optimization of the edge brightening parameter- by alleviating the tendency of the aperture of impinging a much higher level of excitation on its edges than in the medium part of its tail. This statement can be understood from the fact that -diversely from [17], where a $Q$ factor of $-18.64 \mathrm{~dB}$ as well as a flat ending of the aperture (fig. 4 in [17]) can be obtained from the direct optimization of the edge brightening of the aperture distribution analyzed- the result here presented for the same $S L L(-25 \mathrm{~dB})$ reports a $Q$ of $-21.18 \mathrm{~dB}$ and a tail of the aperture which is descending up to its edge.

Although, this proposed technique could be extended to other distributions, the spirit of the present work is based on the idea of using circular Taylor distributions because of they are optimal in terms of efficiency for a desired $S L L$. Additionally, it can be also highly remarkable that an analogous procedure could be easily implemented for linear Taylor distributions.

\section{APPENDIX}

Accuracy of the approximation: Regarding the precision of the $Q$ ratio expressions based on aperture integrals by means the Parseval identity it is necessary to outline the performance of this approximated expression and its level of accuracy versus the formal infinity integral of the far field pattern.

This can be done by simply comparing two different performances: the calculation of the method by integrating the exact expressions of the far field pattern generated by the aperture -on the denominator of (3)- and the method here developed and based on the use of the integral of the aperture -on the denominator of (4). The results regarding this comparison in the worst scenario $\left(S L L_{d}=-25 d B\right)$ are shown in Fig. 9.A.

As an example and due to the fact that it represents the worst scenario in terms of agreement between the results of both methods, the performance of the case for a $S L L_{d}=$ $-25 d B$ has been taken into account. By means of the far field integral in the invisible region, the differences between the computation times of both strategies are reported in Fig. 9.B. It can be appreciated how, as it could be expected, the greater the $\bar{n}$ parameter is, the more computation time will need. The codes to calculate the integrals have been developed in MATLAB [29], more concretely, they have been run in MatlabR2016b. All the computation times are referred to a 


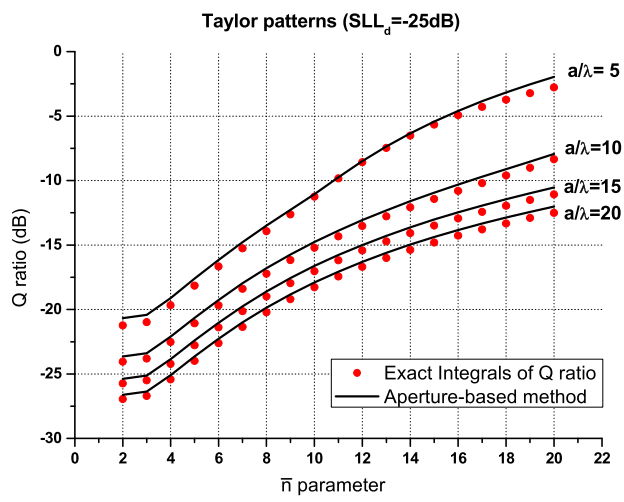

A

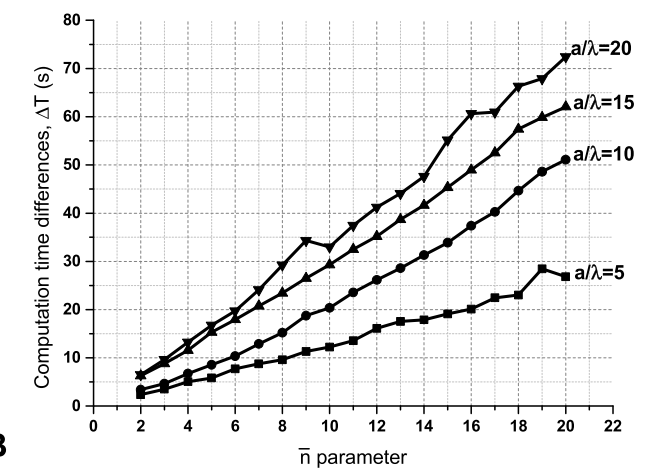

FIGURE 9. Comparison between the exact method for $Q$ calculations and the aperture-based approximation for different radii of the aperture which generates a circular Taylor pattern with a nominal SLL of $-25 \mathrm{~dB}$ : (A) performance, (B) difference between computation times of the codes.

machine with a processor Intel $i 7-2600 \mathrm{CPU} @ 3.40 \mathrm{GHz}$ and a RAM memory of 8GB.

Regarding the level of accuracy we can confirm that the maximum level of misalignment between the 2 methods (the exact and the approximated) for the cases studied in Sect. IV is of 0.002 in natural units (more concretely in the case of $S L L_{d}=-25 d B, \bar{n}=5$ ). In this concrete case it represents an $11.50 \%$ of the nominal value in natural units. For cases of $S L L_{d}=-30 d B, \bar{n}=8$ and $S L L_{d}=-35 d B, \bar{n}=13$ are $7.78 \%$ and $3.67 \%$ respectively.

\section{REFERENCES}

[1] D.-W. Duan and Y. Rahmat-Samii, "A generalized three-parameter (3P) aperture distribution for antenna applications," IEEE Trans. Antennas Propag., vol. 40, no. 6, pp. 697-713, Jun. 1992, doi: 10.1109/ 8.144605.

[2] L. J. Chu, "Physical limitations of omni-directional antennas," $J$. Appl. Phys., vol. 19, no. 12, pp. 1163-1175, Dec. 1948, doi: 10.1063/ 1.1715038

[3] D. R. Rhodes, "On a fundamental principle in the theory of planar antennas," Proc. IEEE, vol. 52, no. 9, pp. 1013-1021, Sep. 1964, doi: 10. 1109/PROC.1964.3231.

[4] D. Rhodes, "On the stored energy of planar apertures," IEEE Trans. Antennas Propag., vol. 14, no. 6, pp. 676-683, Nov. 1966, doi: 10.1109/TAP 1966.1138801

[5] R. C. Hansen, "Fundamental limitations in antennas," Proc. IEEE, vol. 69, no. 2, pp. 170-182, Feb. 1981, doi: 10.1109/PROC.1981.11950.

[6] G. A. Thiele, P. L. Detweiler, and R. P. Penno, "On the lower bound of the radiation q for electrically small antennas," IEEE Trans. Antennas Propag., vol. 51, no. 6, pp. 1263-1269, Jun. 2003, doi: 10.1109/ TAP.2003.812254.
[7] B. L. G. Jonsson, S. Shi, L. Wang, F. Ferrero, and L. Lizzi, "On methods to determine bounds on the $Q$-factor for a given directivity," IEEE Trans. Antennas Propag., vol. 65, no. 11, pp. 5686-5696, Nov. 2017, doi: 10 1109/TAP.2017.2748383.

[8] K. Schab, L. Jelinek, M. Capek, C. Ehrenborg, D. Tayli, G. A. E. Vandenbosch, and M. Gustafsson, "Energy stored by radiating systems," IEEE Access, vol. 6, pp. 10553-10568, Feb. 2018, doi: 10.1109/ ACCESS.2018.2807922.

[9] M. Gustafsson and M. Capek, "Maximum gain, effective area, and directivity," IEEE Trans. Antennas Propag., vol. 67, no. 8, pp. 5282-5293, Aug. 2019, doi: 10.1109/TAP.2019.2916760.

[10] D.-H. Kwon and D. M. Pozar, "Energy storage and radiation $Q$ of infinite planar dipole phased arrays," IEEE Trans. Antennas Propag., vol. 62, no. 1, pp. 153-162, Jan. 2014, doi: 10.1109/TAP.2013.2287515.

[11] A. Ludvig-Osipov and B. L. G. Jonsson, " $Q$-factor and bandwidth of periodic antenna arrays over ground plane," IEEE Antennas Wireless Propag. Lett., vol. 19, no. 1, pp. 158-162, Jan. 2020, doi: 10.1109/LAWP. 2019.2956420.

[12] A. Ludvig-Osipov and B. L. G. Jonsson, "Stored energies and Q-factor of two-dimensionally periodic antenna arrays," IEEE Trans. Antennas Propag., vol. 68, no. 8, pp. 5989-6002, Aug. 2020, doi: 10.1109/TAP.2020. 2979482.

[13] R. C. Hansen, "Apertures," in Microwave Scanning Antennas: A Comprehensive Treatment of Phased Arrays and Quasi-Optical Antennas, R. C. Hansen, Ed. Los Altos, CA, USA: Peninsula Publishing, 1985, ch. 1.

[14] T. T. Taylor, "Design of line-source antennas for narrow beamwidth and low side lobes," Trans. IRE Prof. Group Antennas Propag., vol. 3, no. 1, pp. 16-28, Jan. 1955, doi: 10.1109/TPGAP.1955.5720407.

[15] C. H. Walter, Traveling Wave Antennas. New York, NY, USA: McGraw-Hill, 1965.

[16] F. Ares, E. Moreno, and S. R. Rengarajan, "Optimization of aperture distributions for sum patterns," Electromagnetics, vol. 16, no. 2, pp. 129-143, Mar. 1996, doi: 10.1080/02726349608908466.

[17] J. A. Rodriguez and F. Ares, "Smooth, efficient real amplitude distributions with no edge brightening for linear and circular near-Taylor sum patterns," Electron. Lett., vol. 34, no. 7, pp. 611-612, Apr. 1998, doi: 10. 1049/el: 19980471.

[18] H. Schrank, "Antenna designer's notebook," IEEE Antennas Propag. Soc. News Lett., vol. 28, no. 4, pp. 30-31, Aug. 1986, doi: 10.1109/MAP.1986. 27869.

[19] F. Ares, A. Vieiro, E. Moreno, and S. R. Rengarajan, "Optimization of aperture distributions for difference patterns," J. Electromagn. Waves Appl., vol. 10, no. 3, pp.383-402, Jan. 1996, doi: 10.1163/ 156939396 X00469.

[20] T. Taylor, "Design of circular apertures for narrow beamwidth and low sidelobes," IRE Trans. Antennas Propag., vol. 8, no. 1, pp. 17-22, Jan. 1960, doi: 10.1109/TAP.1960.1144807.

[21] R. S. Elliott, Antenna Theory and Design, Revised ed. New York, NY, USA: Wiley, 2003.

[22] IEEE Standard for Definitions of Terms for Antennas, IEEE Std 145-2013, (Revision of IEEE Std 145-1993), Mar. 2014, pp. 1-50, doi: 10.1109/ IEEESTD.2014.6758443.

[23] R. C. Rudduck, D. C. F. Wu, and R. F. Hyneman, "Directive gain of circular Taylor patterns," Radio Sci., vol. 6, no. 12, pp. 1117-1121, Dec. 1971, doi: 10.1029/RS006i012p01117.

[24] P. Rocca, M. Benedetti, M. Donelli, D. Franceschini, and A. Massa, "Evolutionary optimization as applied to inverse scattering problems," Inverse Problems, vol. 25, no. 12, pp. 1-41, 2009, doi: 10.1088/0266$5611 / 25 / 12 / 123003$

[25] A. Trastoy and F. Ares, "Placing quasi-nulls in planar and conformal arrays," Electromagnetics, vol. 19, no. 4, pp. 373-383, Jul. 1999, doi: 10. 1080/02726349908908654.

[26] W. H. Press, W. T. Vetterling, S. A. Teukolsky, and B. P. Flannery, Numerical Recipes in C, 2nd ed. New York, NY, USA: Cambridge Univ. Press, 1992.

[27] S. Kirkpatrick, C. D. Gelatt, and M. P. Vecchi, "Optimization by simulated annealing," Science, vol. 220, no. 4598, pp. 671-680, May 1983, doi: 10. $1126 /$ science. 220.4598 .671

[28] J. A. Nelder and R. Mead, "A simplex method for function minimization," Comput. J., vol. 7, no. 4, pp. 308-313, Jan. 1965, doi: 10.1093/comjnl/7. 4.308.

[29] The Mathworks. MATLAB R2016b. Accessed: Jul. 17, 2020. [Online]. Available: https://uk.mathworks.com/products/matlab.html 


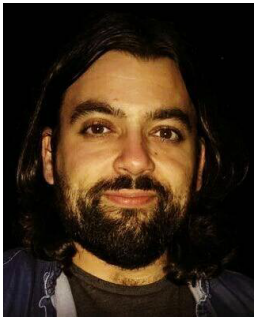

AARÓN ÁNGEL SALAS-SÁNCHEZ was born in A Coruña, Spain, in 1988. He received the physics degree, the M.S. degree in engineering mathematics, and the Ph.D. degree in mathematical modeling and numerical simulation in engineering and applied science from the University of Santiago de Compostela, Spain, in 2012, 2014, and 2018, respectively.

He was a visiting Ph.D. Student with the Department of Electrical Engineering and Information Technology, University Federico II of Naples, Italy, in 2015. He is currently a Xunta de Galicia International Postdoctoral Fellow with the Department of Applied Physics, University of Santiago de Compostela, which brings him the opportunity to be a Visiting Postdoctoral Researcher with the ELEDIA Research Center, University of Trento, Italy, by two years. His main research interests are focused on numerical methods in solving electromagnetic problems and antenna array pattern synthesis.

Dr. Salas-Sánchez has been awarded the Extraordinary Doctorate Award 2017-2018 from the University of Santiago de Compostela for the research work developed during his Ph.D. Thesis.

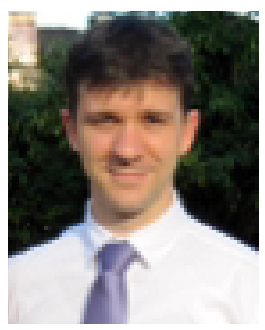

PAOLO ROCCA (Senior Member, IEEE) received the M.S. degree (summa cum laude) in telecommunications engineering in 2005, and the $\mathrm{Ph} . \mathrm{D}$. degree in information and communication technologies from the University of Trento, Italy, in 2008

He was a visiting Ph.D. Student with the Pennsylvania State University, USA, and the University Mediterranea of Reggio Calabria, Italy, in 2012. He was a Visiting Researcher with the Laboratoire des Signaux et Systèmes, L2S@ Supèlec, France, in 2013. He was an Invited Professor with the University of Paris Sud, France, in 2015, and the University of Rennes 1, France, in 2017. He is currently an Associate Professor with the Department of Information Engineering and Computer Science, University of Trento, and a Visiting Professor with the Xidian University, Xi' an, China. He is author/coauthor of one book chapter and of more than 125 journals and 270 conference papers. His main interests include the framework of artificial intelligence techniques as applied to electromagnetics, antenna array synthesis and design, and electromagnetic inverse scattering. He is also a Board Member of the ELEDIA Research Center. In April 2017, he received the National Scientific Qualification for the position of Full Professor. He has been awarded with the Best Ph.D. Thesis Award IEEE-GRS Central Italy Chapter by the IEEE Geoscience and Remote Sensing Society and the Italy Section. He served as an Associate Editor for the IEEE Antennas and Wireless Propagation Letters from 2011 to 2016, IEEE Antennas and Propagation Magazine, and the Microwave and Optical Technology Letters.

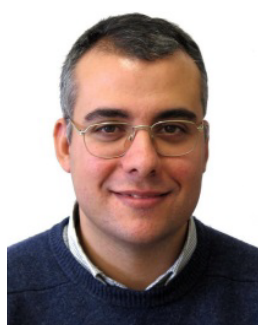

JUAN ANTONIO RODRÍGUEZ-GONZÁLEZ (Senior Member, IEEE) was born in Orense, Spain, in 1972. He received the B.S. and M.S degrees in physics degrees from the University of Santiago de Compostela, Spain, in 1995 and 1996, respectively, and the Ph.D. degree from the University of Santiago de Compostela, in 1999.

$\mathrm{He}$ is currently an Associate Professor with the Department of Applied Physics, University of Santiago de Compostela. He has authored more than 200 articles for journals, conferences, and collaborative volumes. His research interests include numerical methods for solving electromagnetic problems, pattern synthesis, computer programming, and software engineering.
Dr. Rodriguez-Gonzalez was a Secretary of the Spanish Committee of the International Union of Radio Science (URSI) from 2011 to 2017. He received the Outstanding Ph.D. Award from Physics Faculty, University of Santiago de Compostela, in 2000 and the Teaching Innovation Prize from the University of Santiago de Compostela, in 2006.

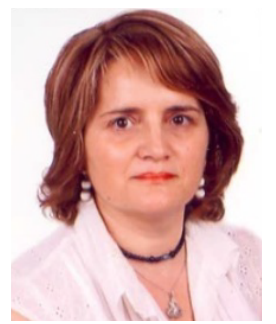

M. ELENA LÓPEZ-MARTÍN was born in Segovia, Spain, in 1963. She received the Ph.D. degree in medicine from the University of Santiago de Compostela, Spain, in 1999.

She is currently an Associate Professor of human anatomy with the Morphological Sciences Department, University of Santiago de Compostela. She has authored or coauthored more than 105 articles and scientific communications. Her current research interests include the study of electromagnetic pollution, the biological effects of mobile telephony, and the therapeutic application of microwaves in the central nervous system (CNS) and peripheral tissues.

Dr. López-Martín has been the Spanish Official Member of the Commission K (Electromagnetics in Biology and Medicine) in the International Union of Radio Science (URSI), since 2013. She serves as a Reviewer for different international journals, including the Progress in Electromagnetic Research (PIER), Bioelectromagnetics, Biomedical and Environmental Science, Mutation Research, and the International Journal of Radiation Biology.

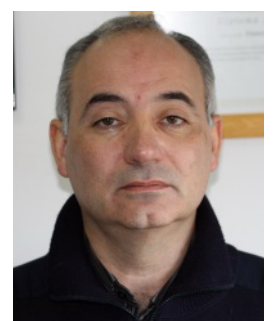

FRANCISCO J. ARES-PENA (Fellow, IEEE) received the B.S., M.S., and Ph.D. degrees in physics from the University of Santiago de Compostela, Spain, in 1986, 1987, and 1993, respectively.

He was a Research Scholar with the Department of Electrical Engineering, University of California, Los Angeles, for two quarters in 1990 and 1991, where he developed the main topic of his Ph. D. thesis. He is currently a Full Professor with the Department of Applied Physics, University of Santiago de Compostela, Spain. He has authored more than 390 papers for journals, conferences, and collaborative volumes. His general research interests include numerical methods in solving electromagnetic problems and antenna array pattern synthesis.

Prof. Ares-Pena served as an Associate Editor for the IEEE Transactions on Antennas and Propagation from 2016 to 2019. He received the Outstanding Ph.D. Award from the Physics Faculty, University of Santiago de Compostela, in 1994, and the Teaching Innovation Prize from the University of Santiago de Compostela, in 2006. He was also the President of the Spanish Committee of the International Union of Radio Science (URSI) from 2011 to 2017. 